\title{
Impulsive Dirac-delta forces in the rocking motion
}

\author{
F. Prieto ${ }^{1, *}$ P.B. Lourenço ${ }^{1}$ and C.S. Oliveira ${ }^{2}$ \\ ${ }^{1}$ Departamento de Engenharia Civil Universidade do Minho \\ Guimaraes. (Portugal) \\ 2 Instituto Superior Tecnico \\ Departamento de Engenharia Civil e Arquitectura. \\ Lisboa. (Portugal)
}

\section{SUMMARY}

In this work the classical theory of one block rocking motion is revisited. A Dirac-delta type interaction as impact mechanism is found to be an alternative for the traditional model. Numerical computations with this new formulation have shown that the agreement with the classical theory is excellent for the case of slender blocks and small displacements. Good agreement with experimental data has also been found for the case of arbitrary angles and slenderness. A probabilistic study of the variations in the coefficient of restitution due to the cyclic degradation at corners during seismic action is also performed.

The approach presented in this paper opens new lines for further theoretical developments and computational applications. Copyright (c) 2002 John Wiley \& Sons, Ltd.

\footnotetext{
${ }^{*}$ Correspondence to: Departamento de Engenharia Civil. Universidade do Minho. Campus de Azurem 4800050. Guimaraes. (Portugal)

mail to: fprieto@civil.uminho.pt
}

Received 
KEY WORDS: ROCKING BLOCK MOTION DIRAC DELTA FORCE

\section{INTRODUCTION}

\section{The Rocking Motion}

Rocking motion (RM) is a dynamical process that occurs frequently in nature. When a rigid body changes instantaneously its center of rotation from one point to another the resulting motion is usually described in terms of differential equations for certain domains of the dynamical variables involved. For simple planar motions, these domains can be referred by the sign of the angular coordinates. Structures which exhibit this behavior were referred by Housner in his pioneer work [1] as "inverted pendulum structures".

Figure 1 shows a block which may start rocking from $\mathrm{O}$ to $\mathrm{O}^{\prime}$ alternatively when an external force is present or certain initial conditions are applied. In spite of the apparently simple nature of the motion, this process hides both a great richness in dynamical behavior and a wide range of practical relevance. In fact, very surprising and interesting peculiarities, such as quasi-periodic and chaotic response or heteroclinic bifurcations among others, have been found for this system.[2],[3],[4],[5].

From a practical point of view, RM is a powerful approach to reveal the dynamic response of monolith columns, rock aggregates, and, in general, most ancient masonry structures [6]. Maintenance and repair of historical buildings has emerged as a recent and active field of interest for the scientific and technical communities with a great importance for the society in general. 
The earthquake hazard, to which architectural heritage is exposed, has proved to be extended and generalized all over the world, being at the same time one of its most devastating agents. The mechanical behavior of the different types of masonry exhibits generally the common feature of a very low tensile strength. This property is so important that the form and dimensions of ancient constructions was determined according to it.

Another relevant feature of masonry components is the high value of the friction coefficient, meaning that conditions for sliding collapse occur less frequently and failure occurs often due to the formation of hinged mechanisms.

The division of the whole body under analysis into rigid blocks, when it is exposed to severe loading, such as earthquakes, is a natural consequence of the hypotheses of zero tensile strength. Therefore, models that consider large rigid assemblies connecting each other by hinges and rocking at these hinges can reproduce the behavior of a large group of masonry constructions under such actions.

The natural frequencies of a structure are key parameters for understanding its seismic response. Due to the mentioned low tensile strength of the masonry, the use of conventional continuum models and analysis tools to obtain eigenfrequencies has to be complemented. In fact, as Housner pointed out, the relevant energy in this case is not the elastic, but the gravitational. As a result, the frequencies are amplitude dependent. This fact leads to a kind of physics qualitatively different from the one present in continuum mechanics; the system behaves essentially as a complex piecewise forced oscillator whose recovery mechanisms are no longer understood in terms of material deformations. The system returns to its equilibrium position by its own weight. 
With respect to the existing works related to RM, there exist a notable division between applied mechanics and earthquake engineering. On one hand, in the fields of mechanical systems with unilateral constraints, discrete dynamical systems and piecewise oscillators, a number of interesting theoretical conclusions have been obtained [7], [8]. On the other hand, in the context of earthquake engineering, although the problem of one block subjected to different earthquake inputs [9], [10] and harmonic forcing [2], [4], [5] has been widely studied, just a very small number of blocks have been taken into account until now [11], [12]. Therefore, an important task and the main motivation of the present work is a formulation that links the theoretical results of applied mathematics with their physical consequences for earthquake engineering.

The paper is organized as follows. In Section 2, the equations of motion for free rocking are revisited. Section 3 introduces the general non-dimensional impact interaction based on Diracdelta forces and verifies their validity by comparison with the linearized model. Then, the form of the forces is extended to arbitrary slenderness and angles, and the response is compared with experimental data. Section 4 presents a probabilistic analysis of the effect of the randomness present in the coefficient of restitution (r). In particular, a model for the cyclic degradation at the block corners during the seismic action is used as a possible source for randomness. Section 5 analyzes the variations of the energy diagrams presented in Section 2. 


\section{EQUATIONS OF MOTION FOR FREE ROCKING}

\section{General dynamic functions}

As shown in Figure 1, and for a movement about center of rotation O, let $\theta$ be the angle that the base of the block forms with the foundation in such a way that $\theta$ decreases counterclockwise. If friction is large enough to prevent sliding, the block will rock around $\mathrm{O}$ and $\mathrm{O}^{\prime}$ changing instantaneously from one center of rotation to the other at every time of impact.

This fact naturally leads to consider two reference systems whose origin is $\mathrm{O}$ and O' (and therefore, two different angular quantities) for each mechanism. Nevertheless, the dynamic variable $\theta$ defined within the reference system with origin at $\mathrm{O}$, can be extended to unify both descriptions of the motion by redefining it as the angle between the foundation and an imaginary line coincident with the base of the block for each rotation.

With this definition, the potential energy is:

$$
U(\theta)=M g(h \cos (\theta) \pm b \sin (\theta))
$$

where the " $\pm "$ sign is referred to the sign of the angle $\theta$ with the assumption that negative values of $\theta$ (counter-clockwise) carry a minus sign. Here, "M" is the mass of the block and "g" the acceleration of gravity.

The kinetic energy for both directions of motion is simply:

$$
T=\frac{1}{2} I \dot{\theta}^{2}
$$

where "I" is the "mass" moment of inertia in relation to one of the corners and $\dot{\theta}$ is the angular velocity. 
In terms of $\alpha$ (see Fig. 1) and the distance from one of the corners to the center of gravity "R", the Lagrangian "L" takes the form:

$$
L(\theta, \dot{\theta})=\frac{1}{2} I \dot{\theta}^{2}-M g R \cos (\theta \mp \alpha)
$$

The Euler-Lagrange equations for $\theta$, regarding each mechanism are:

$$
I \ddot{\theta}-M g R \sin (\theta \mp \alpha)=Q_{\theta}
$$

where $Q_{\theta}$ is a generalized force to be introduced into the problem representing the impact. When the system is set free in rocking motion, the only forces present are the weight of the block and the impacts.

There are four sources of nonlinearity in these equations: (1) the transition from one governing equation to the other at impact times; (2) the impact energy dissipation which induces a jump discontinuity in the angular velocity; (3) the trigonometric term; and, (4) the possible nonlinearities associated to the form of $Q_{\theta}$.

\section{Slender blocks}

The potential energy can be expanded as a Taylor series in $\theta$ for each direction. Assuming a high slenderness $(\cos (\alpha) \approx 1$ and $\sin (\alpha) \approx \alpha)$ and keeping only the terms until second order in $\theta$, the potential energy "U" can be recast as:

$$
U(\theta)=M g R \cos (\theta \mp \alpha) \approx M g R\left[1 \pm \alpha \theta-\frac{\theta^{2}}{2}\right]
$$

Eliminating the constant term equal to $\mathrm{MgR}$, the resulting Lagrangian is:

$$
L=\frac{1}{2} I \dot{\theta}^{2}-M g R\left[-\frac{\theta^{2}}{2} \pm \alpha \theta\right]
$$


In the following, a non-dimensional angle " $\mathrm{x}$ " and a non-dimensional time $\tau$ are adopted:

$$
\begin{aligned}
x & \equiv \frac{\theta}{\alpha} \\
\tau & \equiv p t
\end{aligned}
$$

where "p" is defined as $p \equiv \sqrt{\frac{M g R}{I}}$.

If $x^{\prime} \equiv \frac{d x}{d \tau}$, the Lagrangian function (divided by $\mathrm{MgR}$ ) of the new variables $x$ and $x^{\prime}$ reads:

$$
L\left(x, x^{\prime}\right)=\frac{1}{2}\left(x^{\prime}\right)^{2}+\frac{1}{2}|x|^{2}-|x|
$$

and the classical piecewise equations of motion obtained by the Euler-Lagrange equation in $x$ read:

$$
\begin{cases}x^{\prime \prime}-x+1=0 & x>0 \\ x^{\prime \prime}-x-1=0 & x<0\end{cases}
$$

It must be noticed that, if no external forces other than gravity are present, the total nondimensional energy of the system is constant and reads:

$$
E=\frac{1}{2}\left(x^{\prime}\right)^{2}-\frac{1}{2}|x|^{2}+|x|
$$

\section{BEHAVIOR AT IMPACT}

\section{Traditional formulation}

The sudden changes of velocity at the point $\theta=0$ can be associated with an impulsive force, as it is demonstrated next. The impact defines the transition from rocking around one edge to rocking around the other, and, therefore, the transition from one governing equation to another.

It is commonly assumed that if $\dot{\theta}^{b}$ and $\dot{\theta}^{a}$ are the velocities just before and after each impact, 
respectively, it follows that:

$$
\frac{\dot{\theta}^{a}}{\dot{\theta}^{b}}=r
$$

were, " $\mathrm{r}$ " is defined as the coefficient of restitution.

While the gravity is a constant and conservative force, impulses act each time the structure goes from one mechanism to another (or when $\theta=0$ ), dissipating energy at fixed rates. As a consequence, the energetic levels for the free rocking system show a discontinuous pattern due to the sudden changes in the kinetic energy at the impact times. Figure 2 presents a typical energy diagram for $\mathrm{r}=0.8$.

Since between two consecutive impacts the mechanical energy remains constant, the different energy levels appear as horizontal lines of length equal to the time between impacts. The height of each level is given by the formula:

$$
E_{N}=E_{0} r^{2 N}
$$

Here, " $E_{0}$ " is the initial energy that can be computed by using Eq. (11) and "N" is the impact counter which also labels the energetic levels. When the block is set free to rock the different states of energy can be understood by a discrete mapping between the levels.

Adding Eq.(12) to Eqs.(10), the classical formulation for the one block rocking problem, in terms of the non-dimensional variables for angle and time $(x, \tau)$, is given by:

$$
\left\{\begin{array}{c}
x^{\prime \prime}-x+1=0 \quad x>0 \\
x^{\prime \prime}-x-1=0 \quad x<0 \\
x^{\prime}\left(\tau^{a}\right)=r x^{\prime}\left(\tau^{b}\right)
\end{array}\right.
$$

where $\tau^{a}$ and $\tau^{b}$ represent the times before and after the impacts, respectively.

Although equations (14) are well known in literature, no mention is found in previous works regarding the form and amplitude of the impulsive force. On the contrary, the interest was 
mainly focused in the nature of $r$.

In first place Housner [1] provided a theoretical value of $r$, under the assumption of angular momentum conservation for point $\mathrm{O}^{\prime}$ just before and after the impact, i.e.

$$
I \dot{\theta}-2 b M R \sin (\alpha) \dot{\theta}=I \dot{\theta}^{\prime}
$$

This assumption gives an expression for $\mathrm{r}$ which depends solely on the geometry of the block, as:

$$
r=1-\frac{3}{2} \sin ^{2}(\alpha)
$$

Several discrepancies have been found between the theoretical value given in Eq.(16) and the experimental determinations of $\mathrm{r}[13]$.

Due to this deviation, it has been suggested either to assume the impact as not perfectly inelastic or to adopt empirical measured values of $\mathrm{r}[14],[15]$.

In [13] a frequency distribution of $\mathrm{r}$ was found with a pattern very similar to a Gaussian distribution with a mean value centered at the theoretical value of $\mathrm{r}$ given by Eq.(16).

These discrepancies, together with the fact that in real structures the form of the blocks deviates arbitrary from a parallelepiped ideal block, inspired the probabilistic approach for $\mathrm{r}$ to be addressed in Section 4.

In this work, a general impulsive force $F_{\delta}$ is proposed without assuming any additional hypotheses for the nature of $\mathrm{r}$. The interaction, considered in its most general form, will be built from adequate requirements and a comparison with both the numerical integration of the classical model and experimental data will prove the validity of the proposed approach. The implementation of this interaction into the model allows to replace Eqs.(14) by the equation:

$$
x^{\prime \prime}-x+\operatorname{sign}(x)=F_{\delta}
$$


Here $" \operatorname{sign}(\mathrm{x}) "$ is the signum function. ${ }^{\dagger}$

Construction of the interaction

There are three basic requirements for the impulse $F_{\delta}$ to be satisfied:

- (1) The force is concentrated either at space or time.

- (2) The force may depend on the velocity in such a way that its effect produces a sudden change in the value of the velocity at the critical point equal to r-times its value.

- (3) The force reduces the velocity for both directions of motion.

In order to fulfill the first requirement and inspired on the fact that the velocity can be recast as a Heaviside Step function, a Dirac- $\delta$ type force is introduced. Dirac- $\delta$ interactions are frequently used in physics and engineering. They are referred to quantities that are highly concentrated either time or space (e.g. densities or nuclear forces).

The Dirac- $\delta$ function is defined by its assigned properties:

$$
\begin{array}{r}
\delta(x)=0 \quad x \neq 0 \\
\int_{-x_{a}}^{x_{b}} f(x) \delta(x) d x=f(0)
\end{array}
$$

Where " $x_{a}$ " and " $x_{b}$ " are two real numbers which may be infinite and $f(x)$ is any well-posed function.

${ }^{\dagger}$ For numerical purposes, $\operatorname{sign}(\mathrm{x})$ can be represented by:

$$
\operatorname{sign}(x)=\lim _{n \rightarrow 0} \tanh \left(\frac{x}{n}\right)
$$


As a special case of Eq.(20):

$$
\int_{-x_{a}}^{x_{b}} \delta(x) d x=1
$$

It is highlighted the fact that Dirac- $\delta$ is not a function in the usual way; it is a distribution or generalized function[16]. The Dirac- $\delta$ can be built by a limiting process of sequences of continuous functions. One of the most common is the exponential sequence:

$$
\delta_{n}(x)=\frac{1}{n \sqrt{\pi}} e^{-\frac{x^{2}}{n^{2}}}
$$

where "n" will be referred as the penalty parameter.

Another example is the impulse function centered at $\tau^{*}$ defined as:

$$
\delta_{n}(\tau)=\left\{\begin{array}{c}
0 \quad ; \tau<\tau^{*}-\frac{n}{2} \\
\frac{1}{n} \quad ; \tau^{*}-\frac{n}{2} \leq \tau \leq \tau^{*}+\frac{n}{2} \\
0 \quad ; \tau>\tau^{*}+\frac{n}{2}
\end{array}\right.
$$

Even though, strictly speaking the limit:

$$
\lim _{n \rightarrow 0} \delta_{n}(x)
$$

does not exist, $\delta(x)$ may be treated consistently in the form: [17]

$$
\int_{-x_{a}}^{x_{b}} \delta(x) f(x) d x=\lim _{n \rightarrow 0} \int_{-x_{a}}^{x_{b}} \delta_{n}(x) f(x) d x
$$

Due to the fact that $\delta(x)$ must be approximated by a limit sequence of continuous functions, special care must be taken with the possible sources of error when integrating the differential equation.

The delicate situation appears when the numerical integrator falls inside an epsilonneighborhood around the time of impact; $\left|\tau-\tau^{*}\right| \leq \epsilon$. The traditional formulation imposes a 
form for the velocity such that:

$$
v(\tau)=\left\{\begin{array}{c}
v_{0} \quad \tau<\tau^{*} \\
v^{\prime}=r v_{0} \quad \tau>\tau^{*}
\end{array}\right.
$$

That is, the initial value $v_{0}$ just before the impact is filtered to the value $v^{\prime}=r v_{0}$ just after the impact. Notwithstanding, there exist some flexibility about which must be the value for the velocity precisely at the time of impact $\tau^{* \ddagger}$ in the sense that $v^{*}$ may be equated to $v_{0}$ or $v^{\prime}$ indifferently.

From the point of view of numerical integration the situation is as follows: when the integration reaches the value close to $\tau^{*}$ the values of $\mathrm{v}$ suffer a finite interaction around $\tau^{*}$. Two parameters became relevant, namely, the used time-step for computation " $\Delta \tau$ " and the penalty parameter "n" for the interaction. Both quantities became related by defining the auxiliary parameter $\mu$;

$$
n \equiv \mu \Delta \tau
$$

It must be noticed that for a fixed integration step, if $\mu<1$, the interaction will have no effect in the numerical integration. Therefore, it is needed that $\mu \geq 1$ and the penalty parameter becomes lower-bounded with respect to the time-step by $n \geq \Delta \tau$.

Figure 3 shows in detail what happens around the time of impact for a Dirac- $\delta$ interaction generated by the exponential sequence of Eq.(22). Using a Fehlberg-Runge-Kutta scheme (RKF) with step $\Delta \tau=0.0001$, the solutions for the differential equation were computed stepby-step and represented by circles. The two solid lines correspond to the theoretical values (using the traditional model) for the velocities just before and after the first impact. The interaction $-\left(0.9+\Delta \tau \ln (r) \delta\left(\tau-\tau^{*}\right)\right)$ proportional to $\delta\left(\tau-\tau^{*}\right)$ was also showed as background

\footnotetext{
${ }^{\ddagger}$ For future reference the velocity at the time of impact will be named $v\left(\tau^{*}\right) \equiv v^{*}$. 
in order to better illustrate the situation.

As shown, the integrator takes certain intermediate values between $v_{0}$ and $v^{\prime}$ due to the effect of a finite interaction. A possible way for making numerical computation and theoretical calculus compatible is by approximating the functions of $v^{*}$ to their mean between the interval $\left[v_{0}, v^{\prime}\right]$. This mean, for an arbitrary function $f\left(v^{*}\right)$ is defined as:

$$
f\left(v^{*}\right) \equiv<f>\equiv \frac{1}{\Delta v} \int_{v_{0}}^{v^{\prime}} f(v) d v
$$

where $\Delta v \equiv v^{\prime}-v_{0}$.

Although a method based on a predictor-corrector model or a variable step could lead to greater efficiency in computing, the simpler fixed step method used in this work managed to accurately reproduce numerical and experimental results. Therefore, from now on,(RKF) with fixed time step will be the numerical method used for the computations.

In order to reveal the form of $F_{\delta}$, the gravity and other possible forces different from impulses are excluded.

Let be a particle coming from $x=-\infty$ to the origin with a constant velocity equal to $v_{0}$. When it reaches the point $x=0$ at time $\tau=\tau^{*}$, it suffers an interaction that results in changing its velocity to the value $v^{\prime}=r v_{0}$, being " $\mathrm{r}$ " is the coefficient of restitution.

-Starting from Newton's second law:

$$
\frac{d^{2} x}{d \tau^{2}}=F_{\delta}
$$

-Allowing $F_{\delta}$ to depend on the velocity and space through the following separation of variables:

$$
F_{\delta}(x, v)=B(v) \delta(x)
$$


-Expressing Eq.(29) by using the known property of the Delta function: ${ }^{\S}$

$$
\delta(x(\tau))=\sum_{j} \frac{\delta\left(\tau-\tau_{j}\right)}{\left|x^{\prime}\left(\tau_{j}\right)\right|}
$$

where the sum is extended over all the zeros $\left(\tau_{j}\right)$ of the function $x(\tau)$; in the present case, there is just one zero that occurs at $\tau=\tau^{*}$.

Then Eq. (29) becomes:

$$
\frac{d^{2} x}{d \tau^{2}}=\frac{B(v(\tau)) \delta\left(\tau-\tau^{*}\right)}{\left|v\left(\tau^{*}\right)\right|}
$$

Now, by taking the Laplace transform "L[ ]" at both sides and assuming also that $v\left(\tau^{*}\right)=v^{*}>0:$

$$
s^{2} X(s)-s x(0)-x^{\prime}(0)=\frac{1}{v^{*}} \mathbf{L}\left[B(v(\tau)) \delta\left(\tau-\tau^{*}\right)\right]
$$

Here, $X(s)$ represents the Laplace transform for $x(\tau)$. But $x^{\prime}(0)=v_{0}$, and if it is also assumed that $x(0)=0$, the following holds ${ }^{\pi}$ :

$$
X(s)=\frac{v_{0}}{s^{2}}+\frac{B\left(v^{*}\right)}{v^{*} s^{2}} e^{-s \tau^{*}}
$$

Now, taking first the limit $\tau^{*} \rightarrow 0$ and then the inverse Laplace transform for both sides of the preceding equation:

$$
x(\tau)=v_{0} \tau+\frac{B\left(v^{*}\right)}{v^{*}} \tau
$$

§Provided that $x(\tau)$ is continuous and differentiable and $x^{\prime}\left(\tau_{j}\right) \neq 0$

ף Notice that $\mathbf{L}\left[B(v(\tau)) \delta\left(\tau-\tau^{*}\right)\right]=B\left(v^{*}\right) e^{-s \tau^{*}}$ has been used, which implies a definition of $\delta\left(\tau-\tau^{*}\right)$ such that:

$$
\int_{0}^{\infty} \delta\left(\tau-\tau^{*}\right) f(\tau) d \tau=f\left(\tau^{*}\right)
$$

But this is consistent with the definition of Eqs. $(19,21)$; since, by using the property of Eq.(31) and naming $-x_{a} \equiv x\left(\tau_{a}\right), x_{b} \equiv x\left(\tau_{b}\right)$, the left-hand-side of Eq.(20) becomes:

$$
\int_{x\left(\tau_{a}\right)}^{x\left(\tau_{b}\right)} \frac{x^{\prime}(\tau)}{\left|x^{\prime}\left(\tau^{*}\right)\right|} \delta\left(\tau-\tau^{*}\right) f(x(\tau)) d x=\int_{\tau_{a}}^{\tau_{b}} \delta\left(\tau-\tau^{*}\right) f(\tau) d \tau
$$

which makes sense with the definition of $\delta(\tau)$ by choosing $x\left(\tau_{a}=0\right)=-x_{a}$ and $x\left(\tau_{b}=\infty\right)=x_{b}$ 
Therefore, the velocity just after the impact is:

$$
\frac{d x}{d \tau}=v^{\prime}=v_{0}+\frac{B\left(v^{*}\right)}{v^{*}}
$$

Or, equivalent:

$$
\Delta v=\frac{B\left(v^{*}\right)}{v^{*}}
$$

At this stage, some additional hypotheses about the form of $B(v)$ must be assumed in order to allow further manipulations. Assuming that $B(v)$ can be written as a power function of $v$ :

$$
B\left(v\left(\tau^{*}\right)\right)=K\left[v\left(\tau^{*}\right)\right]^{m}
$$

being " $\mathrm{K}$ " and "m" any two real constants. Then:

$$
K=\Delta v\left[v\left(\tau^{*}\right)\right]^{1-m}
$$

As already mentioned, the numerical integration will take some auxiliary values between $v_{0}$ and $v^{\prime}$. Therefore, functions of $v^{*}$ may be approximated by taking their mean values defined according to Eq.(28). Then, $\left[v\left(\tau^{*}\right)\right]^{1-m}$ is substituted by $<[v]^{1-m}>$ in Eq.(39), leading to:

$$
K=\Delta v<[v]^{1-m}>=\int_{v_{0}}^{v^{\prime}} v^{1-m} d v
$$

This last result implies that:

$$
\left(v^{\prime}\right)^{(2-m)}-\left(v_{0}\right)^{(2-m)}=(2-m) K
$$

If the classical linear relation between the velocities before and after the impact is intended to be satisfied, the preceding formula leads to:

$$
\left[r^{2-m}-1\right] v_{0}^{(2-m)}=(2-m) K
$$

But the left-hand-side of this equation is a function of $v_{0}$ equated to a constant. The only way to fulfil the preceding is that both sides become zero. This leads to $\mathrm{m}=2$, and, therefore:

$$
B(v)=K v^{2}
$$


In order to determine the constant $\mathrm{K}$, the value $\mathrm{m}=2$ is introduced into Eq.(29):

$$
\frac{d v}{d \tau}=K v^{2} \delta(x)
$$

Introducing the relation $\frac{d v}{d \tau}=\frac{d v}{d \tau} \frac{d x}{d x}=\frac{d v}{v d x}$ :

$$
d v=K v \delta(x) d x
$$

Integrating in velocities over $v_{0}$ and $v^{\prime}$ and in space between $-x_{a}$ and $x_{b}$ :

$$
\int_{v_{0}}^{v^{\prime}} \frac{d v}{v}=K \int_{-x_{a}}^{x_{b}} \delta(x) d x
$$

From this result, it is possible to deduce:

$$
K=\ln \left(\frac{v^{\prime}}{v_{0}}\right)
$$

But, since $v^{\prime}=r v_{0}$, the following relation must hold:

$$
K=\ln (r)
$$

And therefore,

$$
F_{\delta}=\ln (r) v^{2} \delta(x)
$$

The given amplitude of the force fulfills the first and second requirements addressed above. Nevertheless, according to the third requirement, it is necessary for the interaction to be sensitive to the direction of motion, in such a way that the force reduces the velocity of the block each time the block hits the horizontal position, for each direction. This requirement can be expressed by:

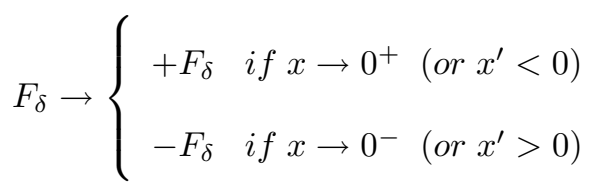

Let the block starts its motion from quiescent initial conditions with a positive value of $x(0)$. The body rotates about center "O" with decreasing $x$. When it is close to $x=0$, it suffers 
an interaction with positive magnitude which reduces its velocity. The block topples with a negative value of $x$ and starts its way back to the origin. Since the amplitude is found to be a power of $\mathrm{v}$ with an even exponent, the interaction experimented by the body now is still positive while it should had been negative in order to decelerate the motion.

This fact results in balancing the effect of the previous interaction and, thus, yielding null damping.

The required behavior for $F_{\delta}$ can be obtained if the factor containing the sign function of the velocity is included.

Therefore:

$$
B(v)=f(v) \operatorname{sign}(v)
$$

or, similarly, another possible choice is by means of the absolute value of $\mathrm{v}$ :

$$
F_{\delta}=K v|v| \delta(x)
$$

Since the constant always gets negative values and in order to highlight the damping nature of the force, "K" will be replaced by A=-K from now on. Assuming that $n<<1$ in the exponential sequence, the explicit form of the interaction is:

$$
F_{\delta}=\frac{-A}{n \sqrt{\pi}} \tanh \left(\frac{v}{n}\right) v^{2} e^{-\frac{x^{2}}{n^{2}}}
$$

Introducing the force found in Eq.(51) into Eq.(17) and by using Eq.(31) results in:

$$
x^{\prime \prime}-x+\frac{|x|}{x}=K x^{\prime}\left|x^{\prime}\right| \sum_{j} \frac{\delta\left(\tau-\tau_{j}\right)}{\left|x^{\prime}\left(\tau_{j}\right)\right|}
$$

This equation represents a piecewise knocked oscillator. The main inconvenient for this approach is that it would imply the impact times to be known quantities, which is clearly unfeasible. 
Instead of following this approach, the Delta will be considered as a function of the space in this work.

Finally, it is noted that, in order to fix a value for the penalty parameter $\mathrm{n}$, the process described next is carried out. In Housner [1] analytical expressions for the times of impact (and in particular for the first one, referenced as $\tau_{1}^{H}$ ) were found. For a given accuracy "q" and timestep " $\Delta \tau "$, " n" can be determined numerically by integrating the differential equation given by Eq.(17) for different values of $\mathrm{n}$ until $\left|\tau_{1}-\tau_{1}^{H}\right| \leq 10^{-q}$, being " $\tau_{1}$ " the first time of impact computed numerically. The penalty parameter must be very small in order to adequately reproduce the desired behavior of the sequence and sign functions. Nevertheless, as already mentioned at the beginning of this subsection, the value of $\mathrm{n}$ must be grater than the time-step. As a consequence $\mathrm{n}$ is limited in its lower value. Numerical integration of Eq.(17) with the force given by Eq.(52) allows to demonstrate that the typical features of rocking motion are reproduced. Figure 4 illustrates the sudden changes in the velocities, due to the impact forces, every time the system crosses at $(x=0)$. This is equivalent to the inclusion of the restitution coefficient in the piecewise equations.

\section{Comparison with classical piecewise model}

Taking the solution for the piecewise formulation in Eq.(10), with initial conditions: $x(0)=x_{0}$ and $\dot{x}(0)=v_{0}$, the equations for each sign of $\mathrm{x}$ are:

$$
\begin{array}{r}
x_{+}(\tau)=1+\left(x_{0}-1\right) \cosh (\tau)+v_{0} \sinh (\tau) \\
x_{-}(\tau)=-1+\left(x_{0}+1\right) \cosh (\tau)+v_{0} \sinh (\tau)
\end{array}
$$

A computer code was written in order to plot the solution of the preceding equations. Since the equations are analytic for each sign of $\mathrm{x}$, the global curve can be built by the addition of 
the solutions $x_{+}$and $x_{-}$with the initial conditions $\left(x_{0}, v_{0}\right)$ updated when $x=0$. The code computes each time of impact and resets the velocities according to the formula:

$$
v\left(\tau_{B}\right)=r v\left(\tau_{A}\right)
$$

The orbits versus time for the conditions $x_{0}=0.5, v_{0}=0$ and $\mathrm{r}=0.7$ are shown in Figure 5 . On top of this diagram, it is possible to observe the points corresponding to the solution of the differential equation of the impact force, for the same initial conditions, the same "r" and with a penalty parameter $n=1 / 5000$. As previously mentioned, for the differential equations an integrator with the algorithm (RKF) was used.

\section{Extending the interaction to the general equations of motion}

The mathematical form of the impact forces for low angles and slender blocks has been outlined in the previous section. The comparison with the traditional formulation has proved the validity of the $F_{\delta}$ proposed. Now, it is mandatory to extend the form of the interaction for arbitrary angles and slenderness, in order to compare the results with experimental data.

In Eq.(4) $Q_{\theta}$ represents the generalized force of the Lagrangian coordinate $\theta$, given by:

$$
Q_{\theta}=\sum_{\nu} \vec{F}^{(\nu)} \cdot \frac{\partial \vec{r}^{(\nu)}}{\partial \theta}
$$

Here, the sum is extended over all the points of the block. In this model, it is considered that all impact forces are normal to the contact surface and act only through the edges. With this assumption $Q_{\theta}$ is simply:

$$
Q_{\theta}=2 b F=2 R \sin (\alpha) F_{\delta}
$$


By using the parameter "p" defined in Eqs. $(7,8)^{\|}$the previous equation becomes:

$$
\ddot{\theta}-p^{2} \sin (\theta \mp \alpha)=p^{2} \sin (\alpha)\left(\frac{2 F}{M g}\right)
$$

Now, for slender blocks and small angles, implementing the non-dimensional variables of Eqs.(7,8), Eq.(59) can be recast as:

$$
x^{\prime \prime}-x \pm 1=\left(\frac{2 F}{M g}\right)
$$

Comparing this last equation with Eq.(17), the following holds:

$$
2 F=M g F_{\delta}
$$

Since the $F_{\delta}$ found in Eq.(51) was expressed in terms of $x$ and $x^{\prime}$, it is necessary to rewrite it using the new variables $\theta$ and $\dot{\theta}$.

For this purpose, it is noticed that $\delta(x)=\alpha \delta(\theta)$ and $x^{\prime}=\frac{\dot{\theta}}{\alpha p}$. Therefore:

$$
F_{\delta}(\theta, \dot{\theta})=\frac{-A}{\alpha p^{2}} \dot{\theta}|\dot{\theta}| \delta(\theta)
$$

Manipulation of Eqs. $(58,61,62)$ results in:

$$
\begin{array}{r}
F=\frac{I}{2 \alpha R}(-A) \dot{\theta}|\dot{\theta}| \delta(\theta) \\
Q_{\theta}=-A I\left(\frac{\sin (\alpha)}{\alpha}\right) \dot{\theta}|\dot{\theta}| \delta(\theta)
\end{array}
$$

Introducing Eq.(64) into Eq.(4), the compact formulation for the rocking motion problem is found:

$$
\ddot{\theta}-p^{2} \sin (\theta \mp \alpha)=-A(r)\left(\frac{\sin (\alpha)}{\alpha}\right) \dot{\theta}|\dot{\theta}| \delta(\theta)
$$

This equation is ready to be compared to experimental data.

\footnotetext{
$\|$ For the case of rectangular blocks, $\mathrm{p}$ is simply $\sqrt{\frac{3 g}{4 R}}$ 
Comparisons with experimental data

Lipscombe [12] is probably one of the best experimental sources for time-angle graphs available in the literature. There, experimental curves for the angles of different blocks as functions of time were presented and discussed. Although the experiments are rather well defined, some relevant facts must be outlined, namely: (1) The blocks used in the experiments were not fully rectangular; and (2) the initial conditions were changed in the numerical model in order to better reproduce the experimental curves.

In Figures 6 and 7, a comparison between experimental and numerical values obtained with the formulation introduced in this work is carried out. As in [12], the initial conditions were adjusted to obtain adequate fitting and the value of $\mathrm{r}$ for the block with $\mathrm{h} / \mathrm{b}=4$ was altered from 0.912 to 0.923 . Under these assumptions good agreement is found.

As it will be discussed in Section 4, a small change in the parameter $\mathrm{r}$ can lead to different solutions. For the block with $\mathrm{h} / \mathrm{b}=4$ the deviation from the value for $\mathrm{r}$ used in our computer code was of the order of 1 per cent. This modification was performed because the blocks used in the experiments were very similar to the one used for the defect analysis, showed in Fig. 8 $[12]$

Regarding the initial conditions, it is worth to stress that if they are allowed to vary, the responses can be significantly modified. The correct parameter-stability analysis of the problem can only be performed in terms of the phase space and parameter stability studies. Several authors, e.g. [2],[4],[6], have determined stability regions in the phase space, which are far outside the scope of the present contribution. Here, the main interest focusses on how the response is qualitatively reproduced using Delta interactions, highlighting at the same time the convenience for a probabilistic treatment of the parameters involved. 


\section{DEFECTS IN BLOCKS}

Possible source for randomness in "r"

During the seismic action, a continuous degradation process of the block interfaces are expected. Moreover, most ancient masonry structures exhibit very weak joints and mortar loss to chemical, physical and mechanical deterioration. As a consequence, the rigid blocks exhibit a rough contour that can modify the instantaneous axis of rotation. The above mentioned "defects" can change significantly the value of $\mathrm{r}$ due to the sensitive nature of the mechanics present in the rocking motion.

Aslam et al. [14] performed a number of experimental and numerical tests on concrete blocks subjected to harmonic as well as to simulated earthquake motions. In that work it was reported that a small change in the value of $\mathrm{r}$ completely changed the time-history response.

Furthermore, it has been noticed by many authors that there is an intrinsic difficulty in obtaining the experimental values of the coefficient $r$ owing to the lack of rigidity of the materials used in the construction. Here, an enhanced model is proposed (see Fig. 8), taking into account the variations of $\mathrm{r}$ when the instantaneous axes of rotation is perturbed by a small movement in the horizontal direction.

Starting with the typical rectangular block of width $2 \mathrm{~b}$ and height $2 \mathrm{~h}$, the moment of inertia is simply $I=\frac{4 M R^{2}}{3}$, where $\mathrm{M}$ is the mass and $\mathrm{R}$ is half the length of the diagonal.

By equating the momentum for point $\mathrm{O}^{\prime}$ just before and after, the impact the classical expression for the coefficient of restitution is obtained:

$$
r=1-\frac{2 M b^{2}}{I}
$$


It is easy to see that the coefficient " $r$ " corresponding to the modified imperfect block given in Figure 8 is:

$$
r^{\prime}=1-\frac{2 M^{\prime} d^{2}}{I^{\prime}}
$$

Now, the non-dimensional imperfection $\epsilon$ is defined by:

$$
\epsilon \equiv \frac{\Delta}{b}
$$

where the imperfection $\Delta$ is given by $\Delta \equiv b-d$. Therefore:

$$
\frac{1-r^{\prime}}{1-r}=\left(1-\frac{\epsilon}{2}\right)^{2}\left(\frac{M^{\prime}}{M}\right)\left(\frac{I^{\prime}}{I}\right)
$$

In the very simple model presented in this work, it will be also assumed that the modifications in the masses and moments of inertia are negligible. That is, $M^{\prime}=M$ and $I^{\prime}=I$.

By performing a translation in the variables with the help of the definitions:

- $\xi^{\prime} \equiv 1-r^{\prime}$

- $\xi \equiv 1-r$

The preceding equation results:

$$
\frac{\xi^{\prime}}{\xi}=\left(1-\frac{\epsilon}{2}\right)^{2}
$$

This study focus on the effect of small displacements in the instantaneous axes of rotation, due to the cyclic degradation at corners when the seismic action takes place. Therefore, it will be assumed that the typical sizes of defect are small compared to the unit value (i.e. $\epsilon \approx 0.05$ ) and all second order terms in $\epsilon$ will be neglected. Under this assumption:

$$
\frac{\xi^{\prime}}{\xi}=(1-\epsilon)
$$

Defining the variation in $\xi$ as:

$$
\Delta \xi=\xi(\epsilon)-\xi
$$


the relative uncertainty in $\xi$ due to the presence of the alteration $\epsilon$ can be computed. This relative uncertainty can be defined as:

$$
\delta \xi \equiv \frac{|\Delta \xi|}{\xi}
$$

Therefore, by means of the preceding equations it is found that ${ }^{* *}$ :

$$
\delta \xi=\epsilon
$$

As $\epsilon$ represents the non-dimensional length of the defect, $\xi$ is found in terms of a quantity with practical relevance. If $\xi$ is considered a random variable with certain 2-parameter distribution of probability, the quantities $\bar{\xi}$ and $\delta \xi$ can be identified with these parameters, namely, the mean value and the variance (or its square root, the standard deviation). By assigning $\delta \xi$ to the dispersion of $\xi$, and due to the linear relationship between the two variables $\mathrm{r}$ and $\xi$ it also holds:

$$
\delta(r)=\delta(\xi)=\epsilon
$$

Therefore, two parameters for a probability distribution in $\mathrm{r}$ can be used; the mean $\bar{r}$ and the dispersion $\sigma(r)$. While the former can be regarded as the theoretical value of the coefficient of restitution for perfectly inelastic impact, the latter is simply the global size of defect $\epsilon$.

Nevertheless, the random variable $\mathrm{r}$ is double-bounded between 0 and 1 , so its associated probability distribution must be truncated at this values, that is, defined piecewise:

$$
f(r)= \begin{cases}0, & r<0 \\ C N_{[\bar{r}, \epsilon]} & , 0 \leq r \leq 1 \\ 0, & r>1\end{cases}
$$

\footnotetext{
${ }^{* *}$ As there is no possible misunderstanding with the Dirac- $\delta$ function, the same symbol $\delta$ was used. 
Here " $N_{[\bar{r}, \epsilon]}$ " is a normal distribution of parameters $\bar{r}$ and $\epsilon$, and "C" is a constant for normalization.

This fact renders the calculus very complex and the physical meaning of the quantities involved is missed. Fortunately, the amplitude of the force A, due to its domain, exhibits more flexibility.

Probability distributions for the interaction magnitude

In [13] a frequency distribution for $\mathrm{r}$ was found in tests of free rocking regime following the Irpina 1980 earthquake. The experimental mean obtained was very close to the theoretical value for perfectly inelastic impact, given by Eq.(16) and the values of $\mathrm{r}$ were significantly scattered through a distribution which was very similar to a Gaussian distribution centered at the theoretical value of $r$.

According to these results and the formulation developed in the previous Section, it will be assumed that the probability distribution in $\mathrm{r}$ behaves as a Gaussian distribution with parameters $\bar{r}$ and $\sigma^{2}(r)$ (where $\bar{r}$ is the mean value of $\mathrm{r}$ and $\sigma(r)=\epsilon$ is the standard deviation). Since $\mathrm{r}$ is regarded as a random variable, $\mathrm{A}(\mathrm{r})=-\ln (\mathrm{r})$ is also considered as given by a probability distribution $\mathrm{g}(\mathrm{A})$. As stressed above, notice that the domains of the variables $\mathrm{r}$ and $\mathrm{A}$ are $[0,1]$ and $[0, \infty)$, respectively. Therefore A takes only positive values and a possible choice for $g(A)$ may be a lognormal distribution of parameters "a" and "b" defined by:

$$
g(A)=\frac{1}{A b \sqrt{2 \pi}} \exp \left[-\frac{1}{2}\left[\frac{\ln (A)-a}{b}\right]^{2}\right]
$$

The mean and the dispersion are known parameters given by:

$$
\begin{array}{r}
<A>=e^{a+\frac{1}{2} b^{2}} \\
\Delta A=e^{a} \sqrt{e^{b^{2}}\left(e^{b^{2}}-1\right)}
\end{array}
$$


Now, the new parameters "a" and "b" for the distribution of A need to be identified with physically meaningful quantities. Since, the sources of information are $\bar{r}$ and $\epsilon$, the values for "a" and "b" will be expressed in terms of them.

Rigorously, this process would imply equating Eqs. $(78,79)$ to:

$$
\begin{array}{r}
<A>=E\{\ln (1 / r)\} \\
\Delta A=E\left\{[\ln (1 / r)-E(\ln (1 / r))]^{2}\right\}
\end{array}
$$

But when changing to variable $\mathrm{r}$, since the integration must be performed between 0 and 1 , the results turn to be very complex. In fact, after some algebraic manipulations it can be shown that:

$$
<A>=-\ln (\bar{r})-R(\bar{r}, \epsilon)
$$

Where $R(\bar{r}, \sigma)$ is a rest defined by:

$$
R(\bar{r}, \epsilon)=\frac{\int_{0}^{1} d z \ln (z) \exp \left[-\frac{1}{2}\left(\frac{\bar{r}(z-1)}{\epsilon}\right)^{2}\right]}{\int_{0}^{1} d z \exp \left[-\frac{1}{2}\left(\frac{\bar{r}(z-1)}{\epsilon}\right)^{2}\right]}
$$

If $D(\bar{r}, \sigma)$ is defined by:

$$
D(\bar{r}, \epsilon)=\frac{\int_{0}^{\frac{1}{\bar{r}}} d z(\ln (z))^{2} \exp \left[-\frac{1}{2}\left(\frac{\bar{r}(z-1)}{\epsilon}\right)^{2}\right]}{\int_{0}^{\frac{1}{\bar{r}}} d z \exp \left[-\frac{1}{2}\left(\frac{\bar{r}(z-1)}{\epsilon}\right)^{2}\right]}
$$

It also holds:

$$
\Delta A=D-R^{2}
$$

Although by continuing with this process would imply handling with integrals of the type showed above leading to expressions with little physical relevance, some interesting conclusions can still be obtained by simple assumptions about the form of $\langle A\rangle$ and $\Delta A$. 
On one hand, numerical integrations of $\mathrm{R}$ for different values of $\bar{r}$ and $\epsilon$ have shown that in fact $\mathrm{R}$ is very small compared to $\ln (1 / \bar{r})$ for $\sigma \leq 0.1$. Therefore, $<A>$ may be approximated by $-\ln (\bar{r})$ for small defects.

On the other hand, from the simple inspection of the relation $\mathrm{A}(\mathrm{r})=-\ln (\mathrm{r})$, it holds:

$$
d A=-\frac{1}{r} d r
$$

From which, in a rough approximation $\delta A \approx\left|\frac{\delta r}{r}\right|$. Then, it can be assumed that:

$$
\begin{array}{r}
<A>=\ln \left(\frac{1}{\bar{r}}\right) \\
\Delta A=\frac{\epsilon}{\bar{r}}
\end{array}
$$

From equations $(78,79)$ and $(87,88)$, the values of the parameters "a" and "b" can be found in terms of " $\epsilon "$ and " $\bar{r} "$ :

$$
\begin{aligned}
& a=\ln \left[\frac{\ln \left(\frac{1}{\bar{r}}\right)}{\sqrt{1+\frac{\epsilon^{2}}{\bar{r}^{2}\left(\ln \left(\frac{1}{\bar{r}}\right)\right)^{2}}}}\right] \\
& b=\sqrt{\ln \left[1+\frac{\epsilon^{2}}{\bar{r}^{2}\left(\ln \left(\frac{1}{\bar{r}}\right)\right)^{2}}\right]}
\end{aligned}
$$

And the probability distribution $\mathrm{g}(\mathrm{A})$ can be stored for future random number generation for the interaction amplitude. 


\section{INFLUENCE OF THE INDETERMINACY OF THE INTERACTION AMPLITUDE ON THE ENERGY DIAGRAMS}

As suggested above, variations in the value of A (or $\mathrm{r}$ ) can lead to significative differences in the response. In particular, as from Eq.(13), it is found that:

$$
E_{N}=E_{0} e^{-2 N A}
$$

Therefore, an exponential decay of the energetic levels occurs with the impacts. If the relative distance " $\gamma$ " between two consecutive levels is defined as:

$$
\gamma \equiv \frac{-\Delta E_{N}}{E_{N}}=\frac{E_{N}-E_{N+1}}{E_{N}}
$$

The following relation holds:

$$
\gamma=1-e^{-2 A}
$$

where $\gamma$ depends solely on A, being independent of level N.

If a block with $r=0.5$ suffers an impact such that this value is altered to $\mathrm{r}=0.8$, its energy diagram will be consequently changed. As shown in Figure 9, it will go from the right diagram levels to the left configuration and its response will be significantly different. The interaction amplitude "A" becomes dependant of $\mathrm{N}$ for a degradation process in general.

An interesting case is when the degradation occurs at each impact and $\mathrm{A}$ is a continuous function of $\mathrm{N}$. The rate of change of A with the impact counter will be a function of the dispersion of A. That is:

$$
\frac{A(N)-A(0)}{N}=f(\Delta A)
$$


Equation (88) provides reasonable approximations for $\Delta A$ and, in principle, any function of $\Delta A$ can be constructed from them. In the simplest case analyzed here it will be assumed that $f(\Delta A)$ is linear with respect to $\Delta A$, so that A decreases proportionally to $\epsilon$. If the constant of proportionality is chosen to be precisely $\bar{r}$, it is found $f(\Delta A)=\bar{r} \Delta A=\epsilon$. And therefore:

$$
A(N)=A(0)+N \epsilon
$$

This model represents a block which is subjected to severe dynamic loading and features material loss at each impact. In Figure 10, for a 5 per cent of degradation, it is shown how this kind of situation may alter significantly the response of the block.

\section{CONCLUSIONS AND OPEN PROBLEMS}

The approach presented in this work provides a significant step towards a rocking motion formulation that can be extended to more complex problems than the solely single block rocking dynamics.

Both the discontinuous nature of the equations and the ad hoc inclusion of a coefficient of restitution difficult the work of generalizing the formalism.

The Dirac-delta force proposed in this work was successful in reproducing both classical formulation and experimental tests. Its implementation into the dynamics is automatic and simplifies the governing system of differential equations.

Although a unique governing equation is still desired in order to generalize the problem, the substitution of the traditional formalism by an interaction is a first step in this direction. 
The energy diagrams presented offer an interesting way for deriving quantities with physical relevance (i.e. energy loss transmitted to the foundation); they also inspired the probabilistic study carried out.

Although the energetic diagram for a given block changes when it is exposed to external loading, it can still be a useful tool by means of considering it as a basic skeleton for the free rocking dynamics. How this diagram changes for arbitrary external forces is an open problem which may highlight important features of the RM process. At this stage, a simple form of linear degradation with impact has been introduced.

It is in the scope of future research to find a unique governing equation for RM which incorporates Delta impact forces plus a generalized seismic loading. If this goal is reached, the possibility for extending the formulation to a higher number of blocks and studying the dynamics by means of statistical mechanics will be opened.

\section{REFERENCES}

1. Housner, W. G. The behavior of inverted pendulum structures during earthquakes. Bull.Seismol.Soc.Amer 1963; 53:403-417.

2. Hogan, S. J. On the dynamics of rigid block motion under harmonic forcing. Proc.Roy.Soc.Lond. A 1989; 425:441-476.

3. Hogan, S. J. Heteroclinic bifurcations in damped rigid block motion. Proc.Roy.Soc.Lond. A 1992; 439:155162.

4. Spanos, P.D., and Koh, A.S. Rocking of rigid blocks due to harmonic shaking. J. Engrg. Mech. Div., ASCE $1984 ; 110$ (11), 1672-1642

5. Yim, S.C.S., and Lin, H. Nonlinear impact and chaotic response of slender rocking objects. J.Engrg.Mech., ASCE 1991; 117:2079-2100. 
6. Augusti, G. and Sinopoli, A. Modelling the dynamics of large block structures. Meccanica 1992;.27:195-211.

7. Leon, M., Martin de Diego, D. On the geometry of non-holonomic Lagrangian systems, J. Phys.,37 (7) (1996), 3384-3414

8. Koon, W.S., Marsden, J.E. The Hamiltonian and Lagrangian approaches to the dynamics of nonholonomic systems. Rep. Math. Phys. 40 (1997), 21-62

9. Yim, C. S., Chopra, A. K. \& Penzien, J. Rocking response of rigid blocks to earthquakes. Earthquake Engineering and Structural Dynamics 1980; 8:565-587.

10. Makris, N. and Roussos, Y. S. Rocking response of rigid blocks under near-source ground motions. Geotéchnique 1999; 50 No. 3;243-262.

11. Psycharis, I. N. Dynamic behavior of rocking two-block assemblies. Earthquake Engineering and Structural Dynamics 1990; 19:555-575.

12. Lipscombe, P. R. Dynamics of rigid block structures. PhD Thesis 1990. University of Cambridge, Cambridge, England.

13. Liberatore, D. et al. Rocking of slender blocks subjected to seismic motion of the base. Proc. 12th Europ. Conf. Earth. Eng. Ref (760), 2002.

14. Aslam, M., Godden, W.G. \& Scalise, D.T. Earthquake rocking response of rigid bodies. J.Struct.Div. 1980; 106(2):377-392.

15. Tso, W. K. and Wong, C.M. Steady state rocking response of rigid blocks to earthquakes. Part 1: Analysis. Part 2: Experiment. Earthquake Engineering and Structural Dynamics 1989; 18:89-120.

16. Kaplan, W. (1991) Advanced Calculus. Fourth Edition. Addison Wesley.

17. Arfken, G.B., and Weber, H.J. (1995) Mathematical Methods for Physicists. Fourth Edition. Academic Press. 


\section{FIGURES}

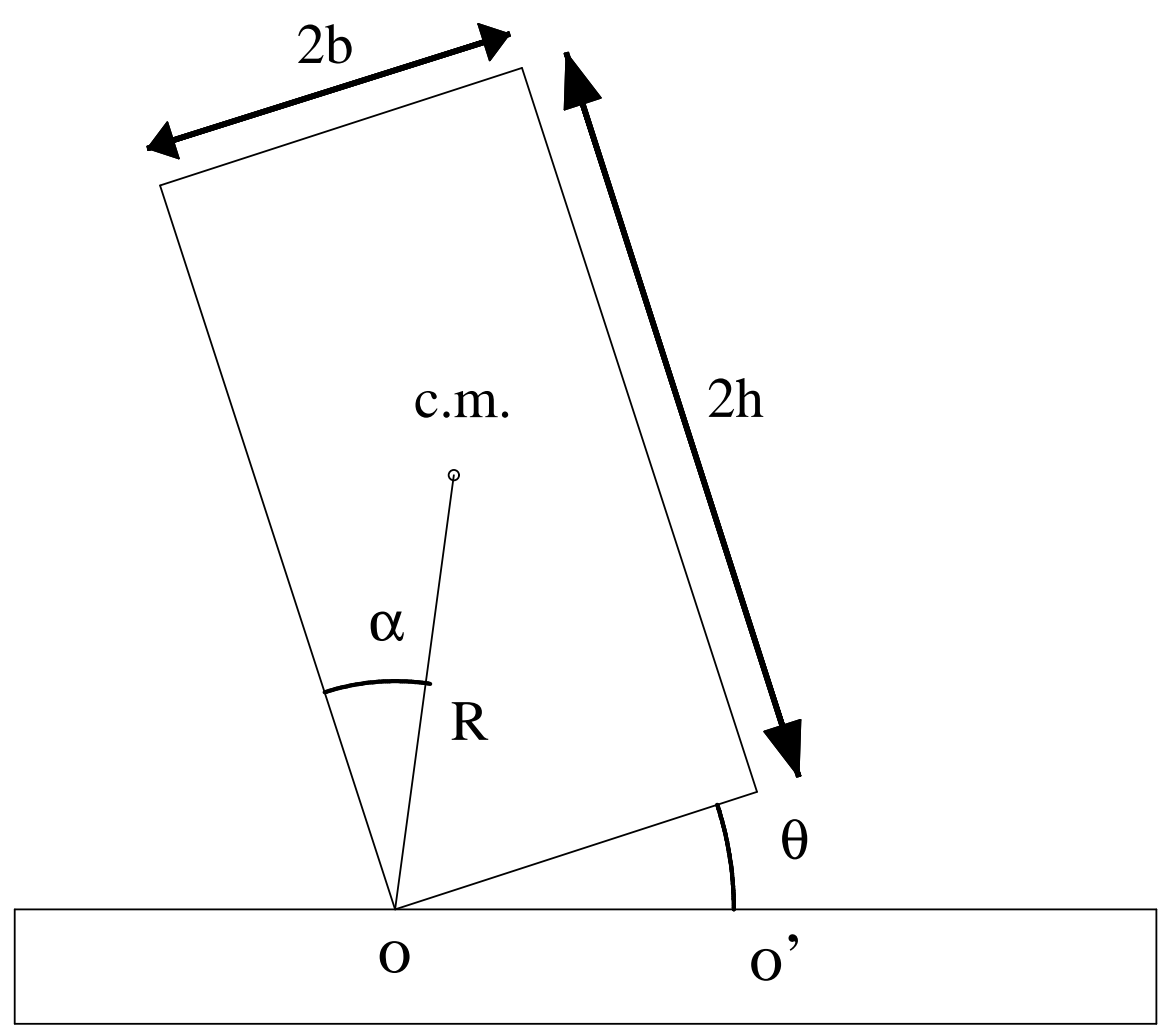

Figure 1. One rocking block - Geometry and notation (c.m. indicates the center of mass) 


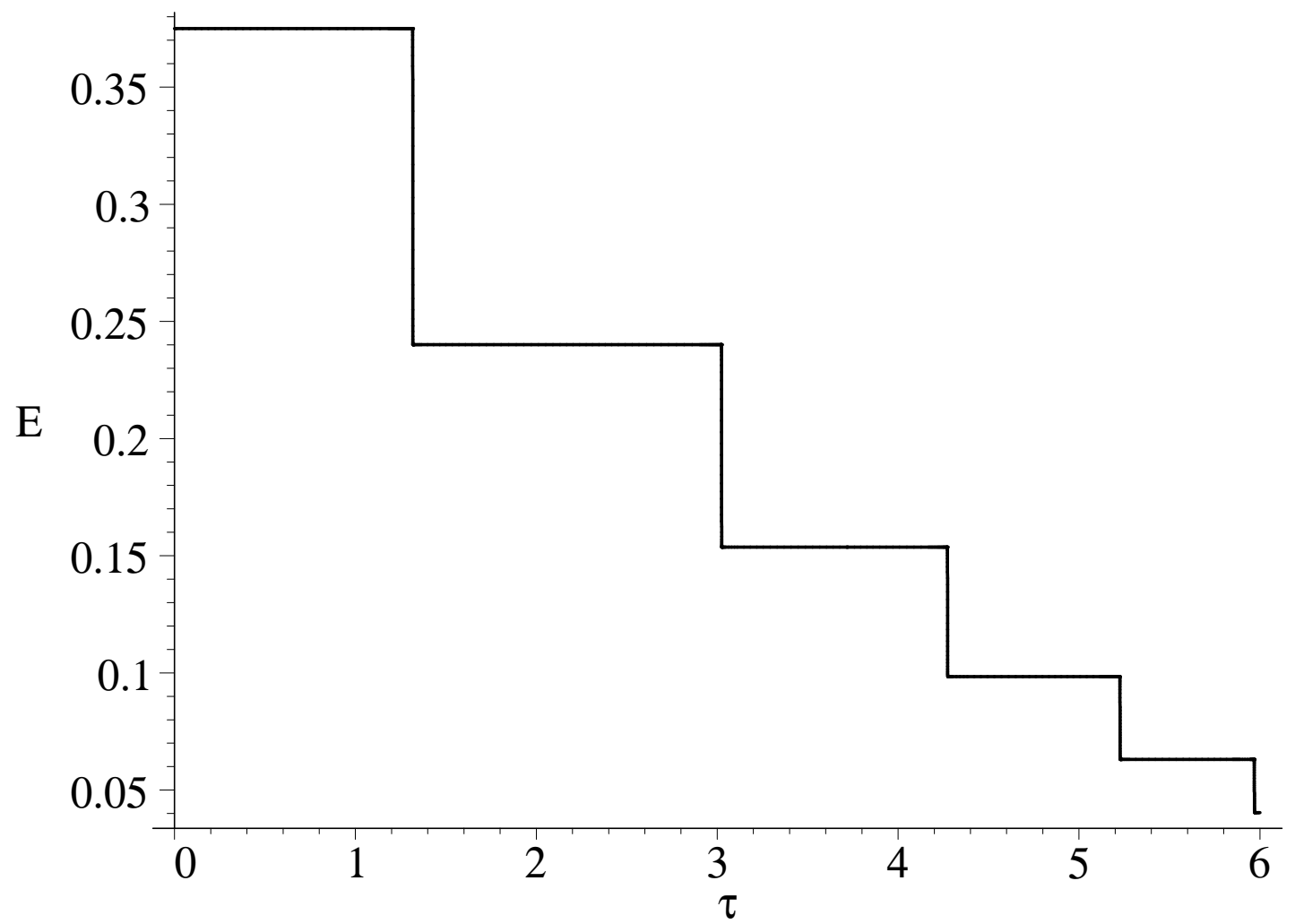

Figure 2. Non-dimensional time and energy for $E_{0}=0.375, \mathrm{r}=0.8$ and initial conditions $\left(x_{0}=0.5\right.$, $\left.v_{0}=0.0\right)$ 


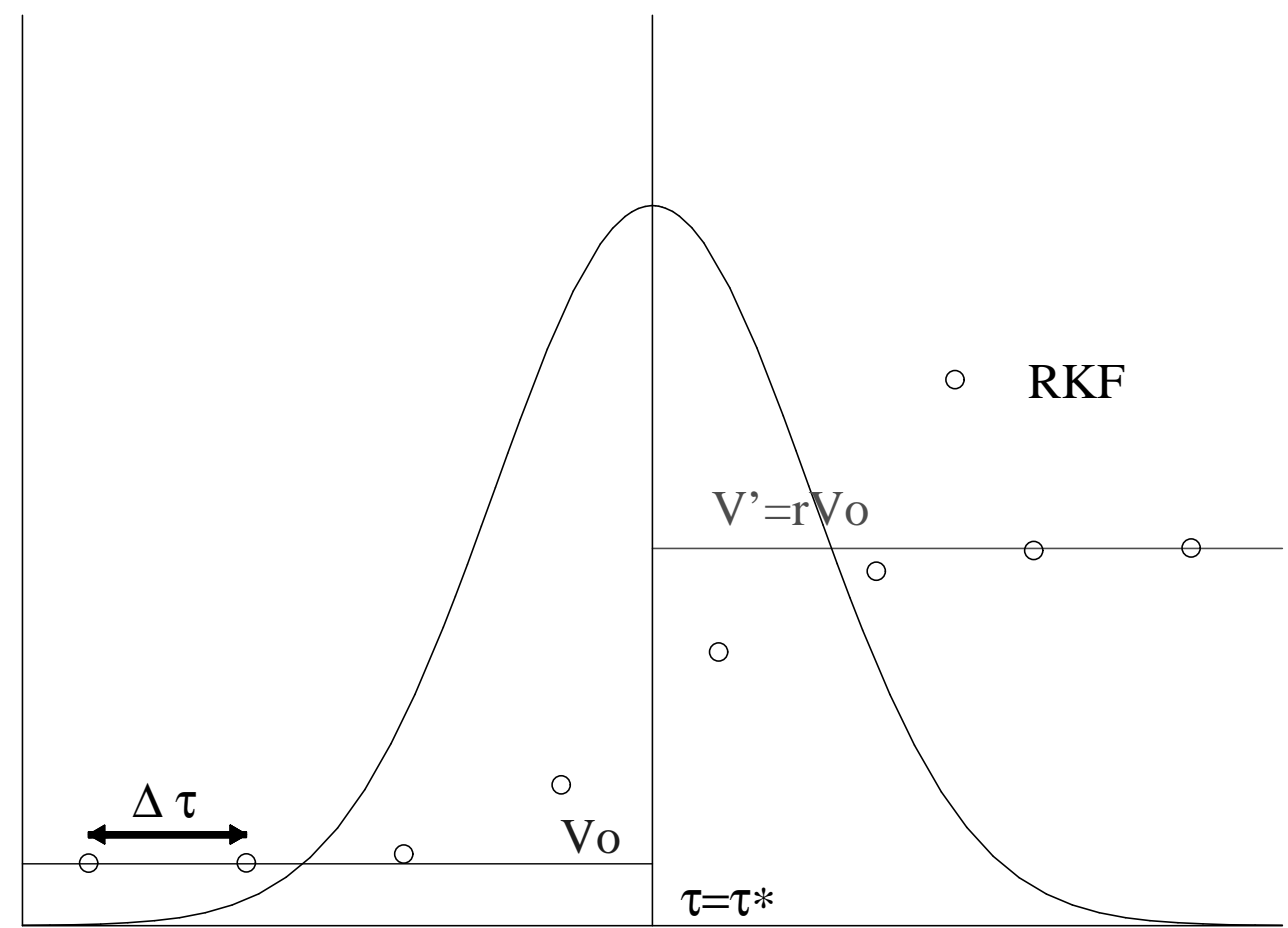

Figure 3. Intermediate values taken by the numerical integration around the impact time as a consequence of a finite interaction. 


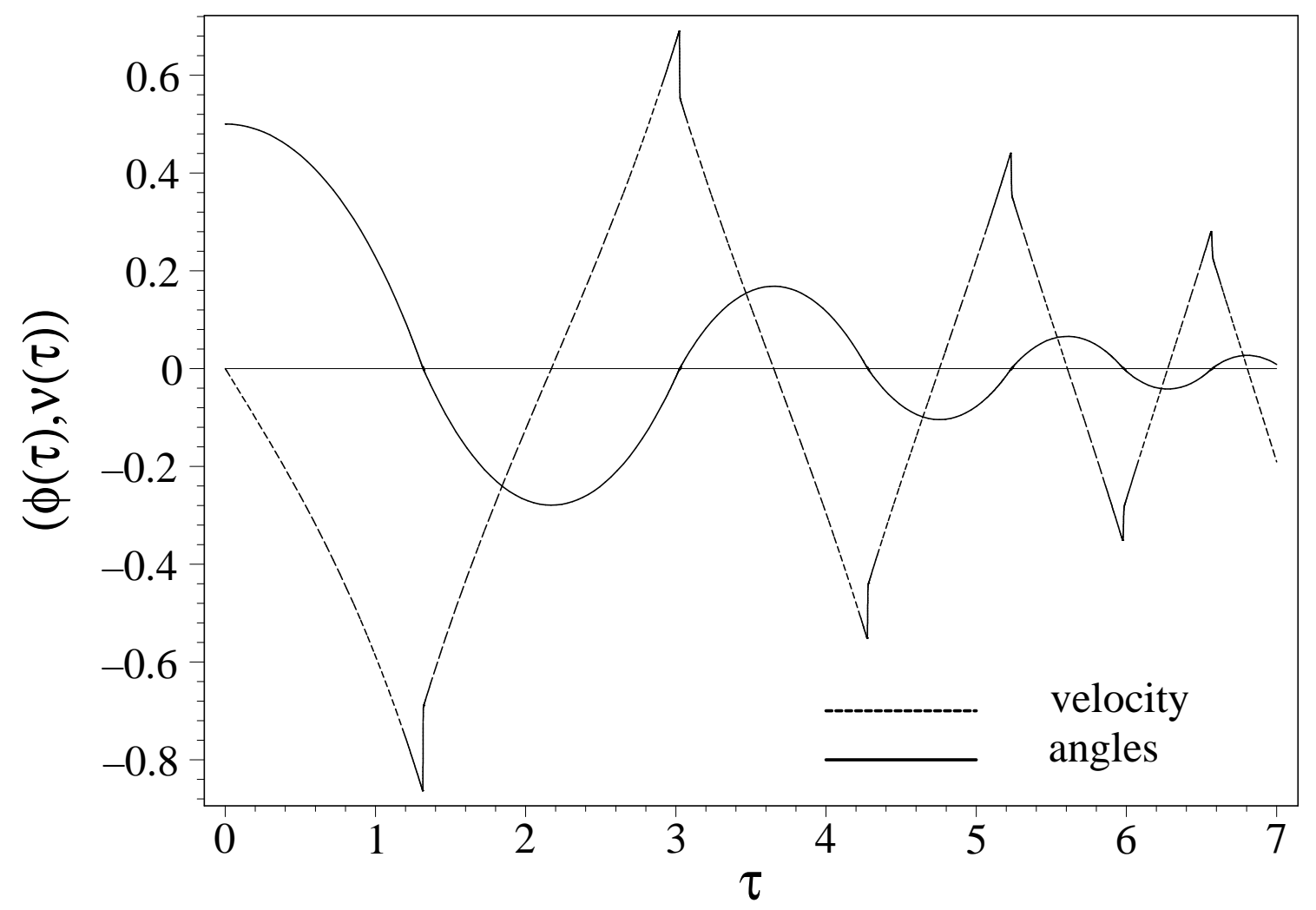

Figure 4. Velocity and orbits for $\mathrm{r}=0.8$ and initial conditions $\left(x_{0}=0.5, v_{0}=0.0\right)$ 


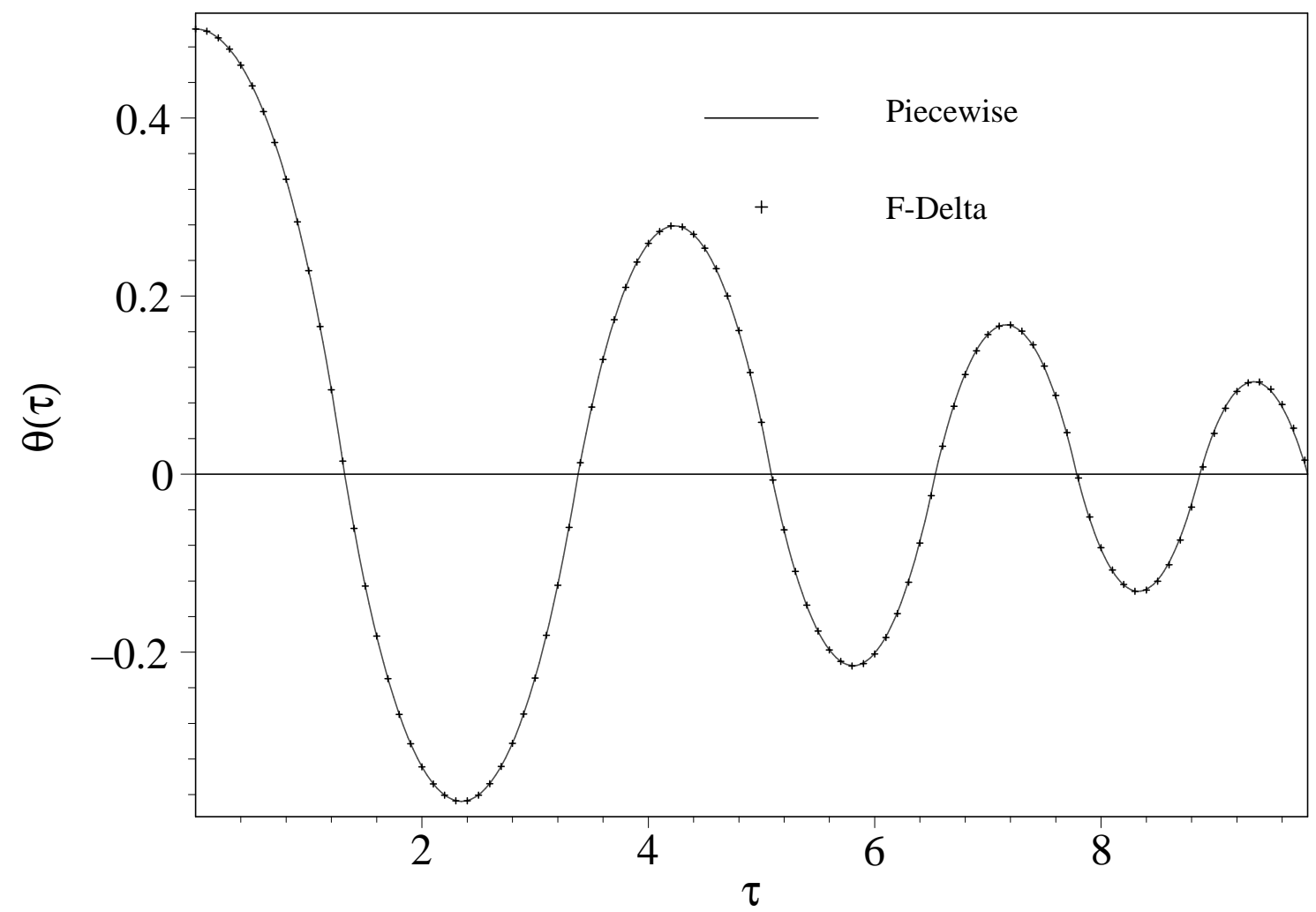

Figure 5. Comparison with piecewise Housner model for $\mathrm{r}=0.8$ and initial conditions $\left(x_{0}=0.5\right.$,

$$
\left.v_{0}=0.0\right)
$$




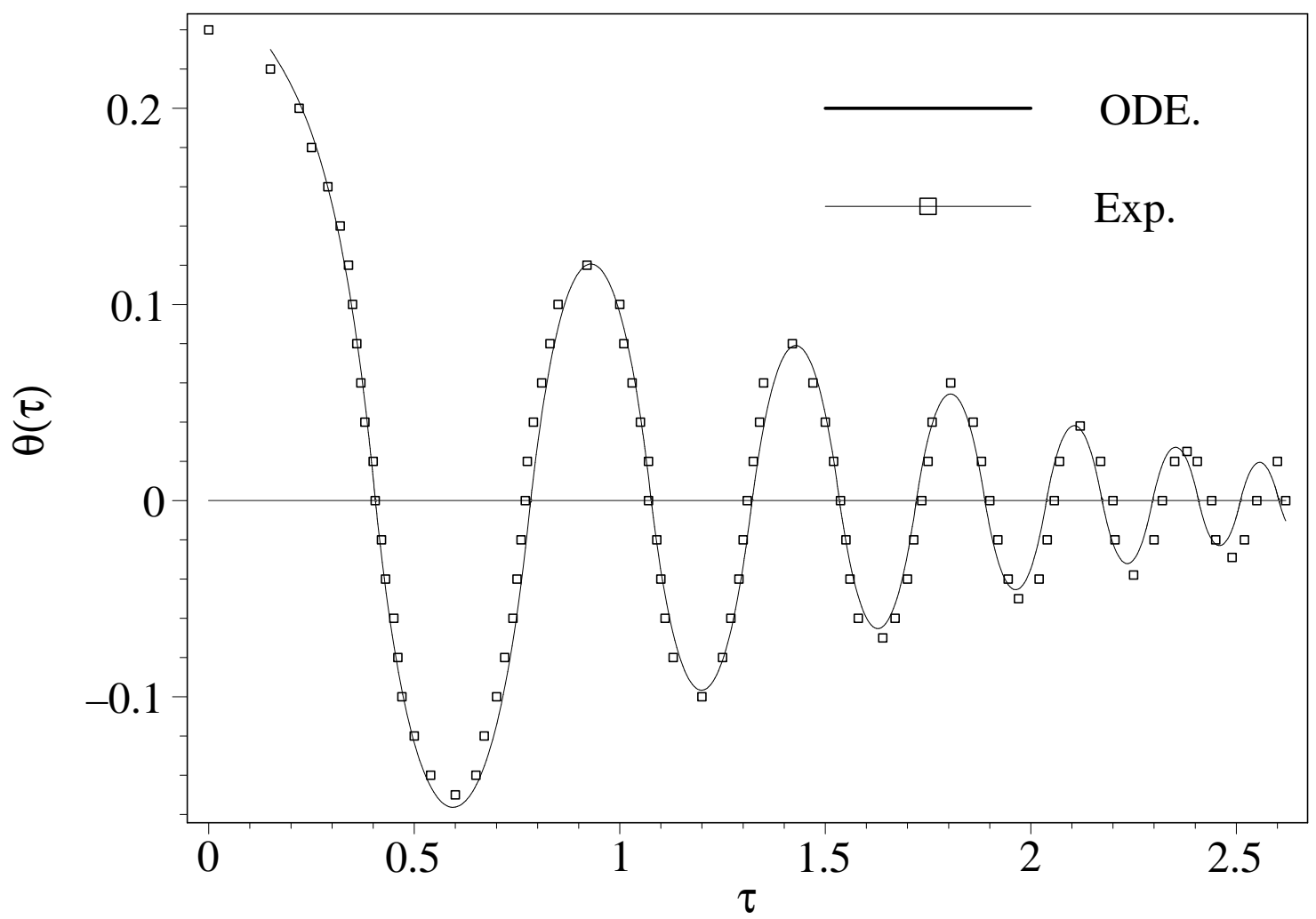

Figure 6. Lipscombe block $\mathrm{h} / \mathrm{b}=4$. Initial conditions: $\theta(0)=0.23, \dot{\theta}(0)=-0.32, \mathrm{r}=0.923$ 


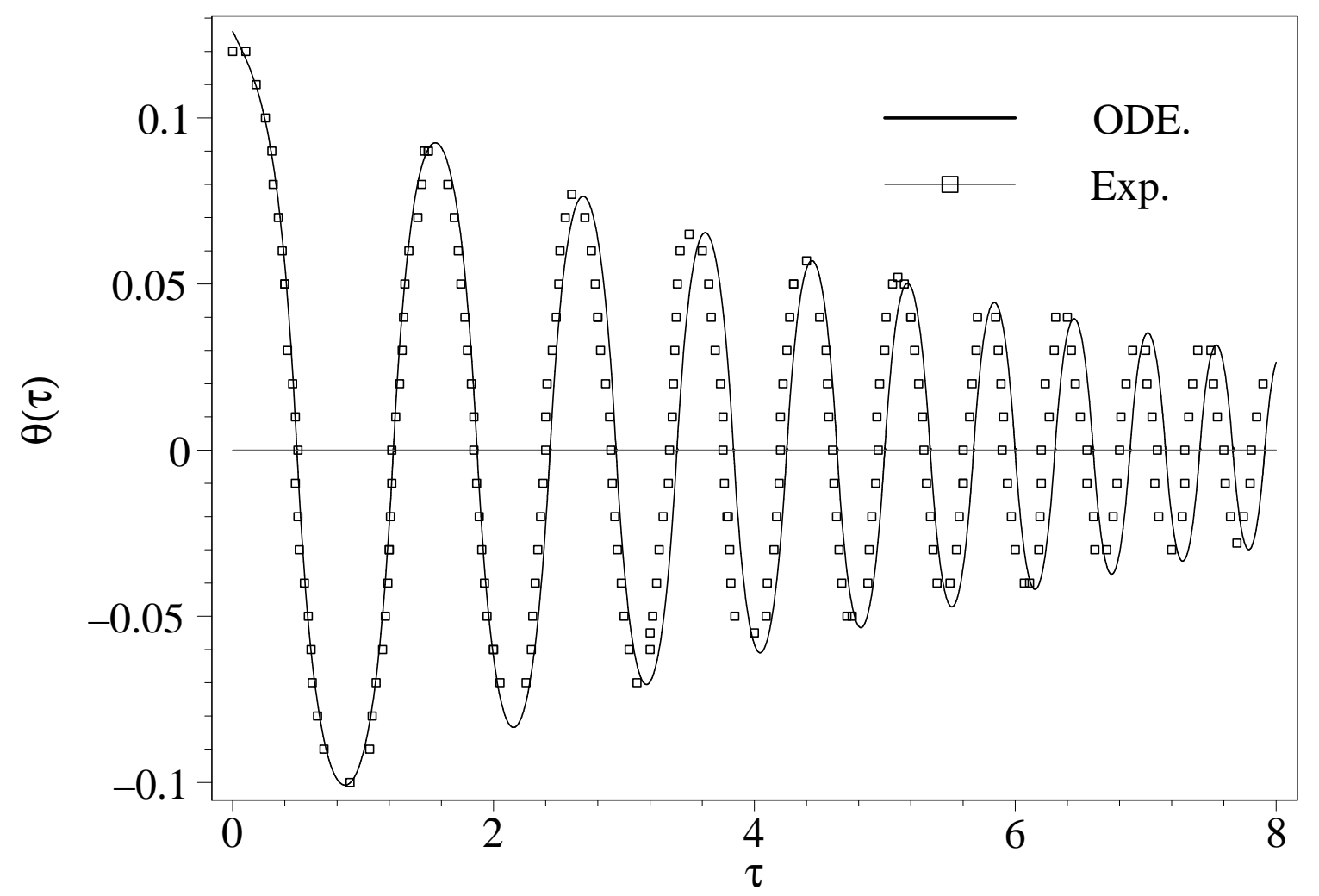

Figure 7. Lipscombe block $\mathrm{h} / \mathrm{b}=8$. Initial conditions: $\theta(0)=0.126, \dot{\theta}(0)=-0.08, \mathrm{r}=0.9769$ 


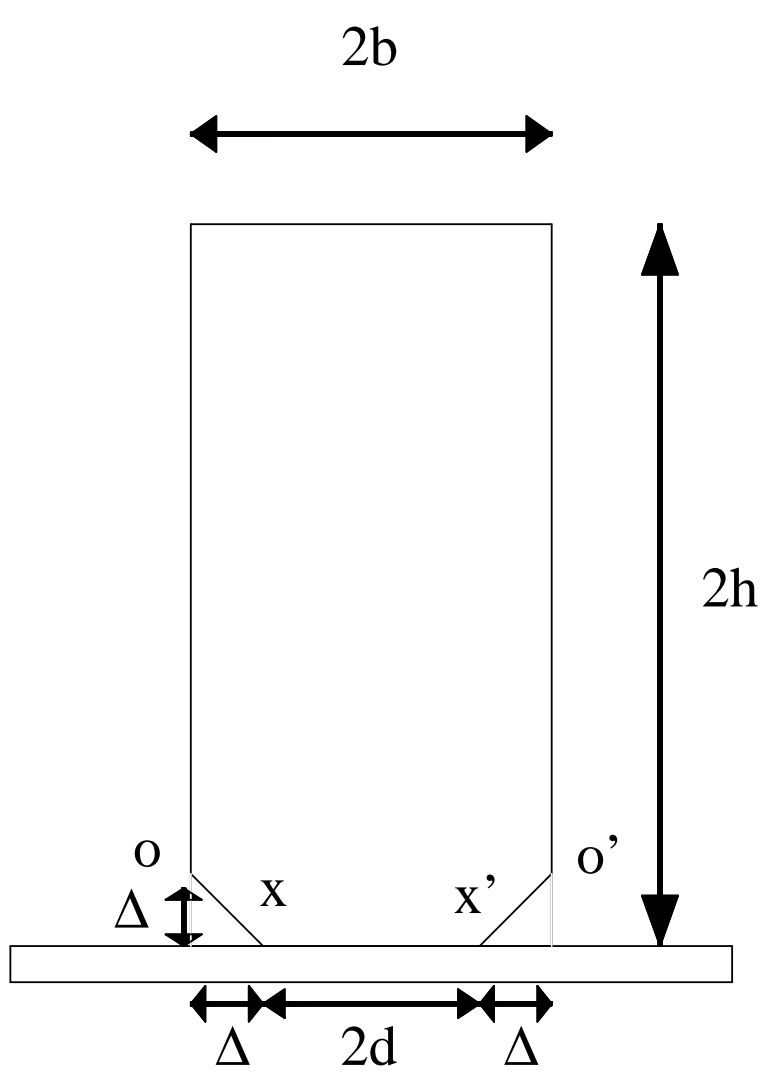

Figure 8. Model of defect. 


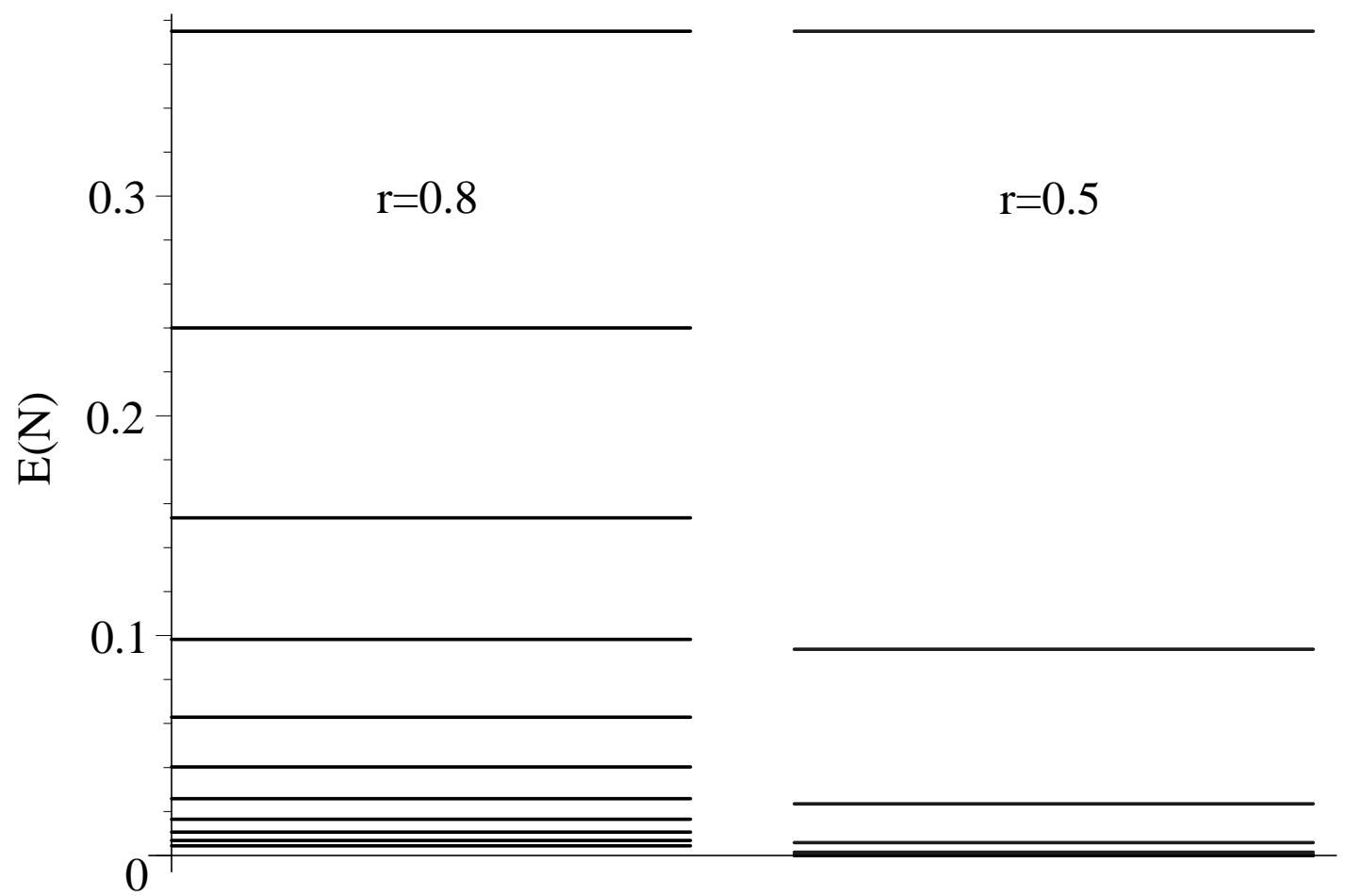

Figure 9. Energy levels for $r=0.5$ and $r=0.8$ 


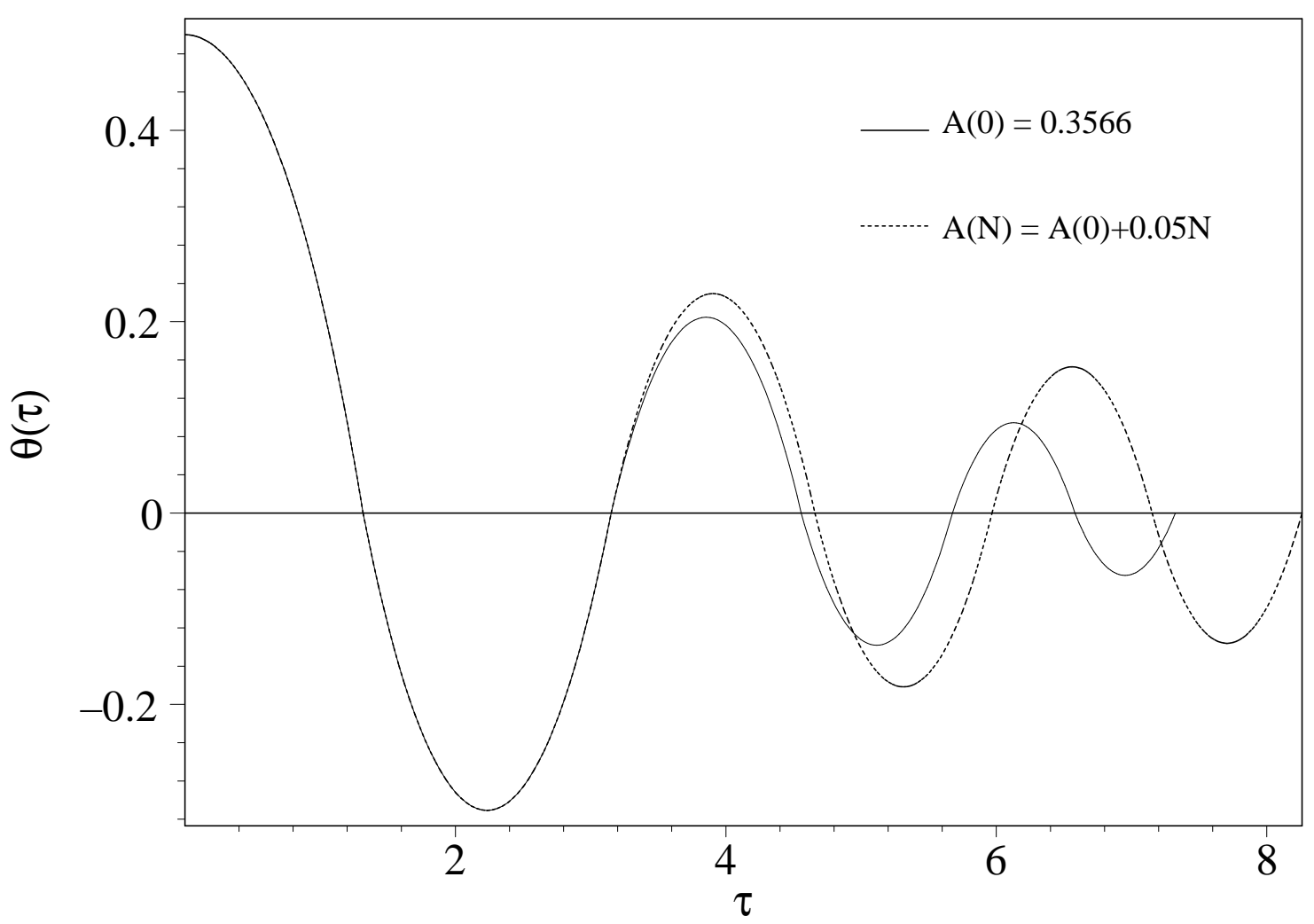

Figure 10. Variation in the orbits for a continuous degradation. 